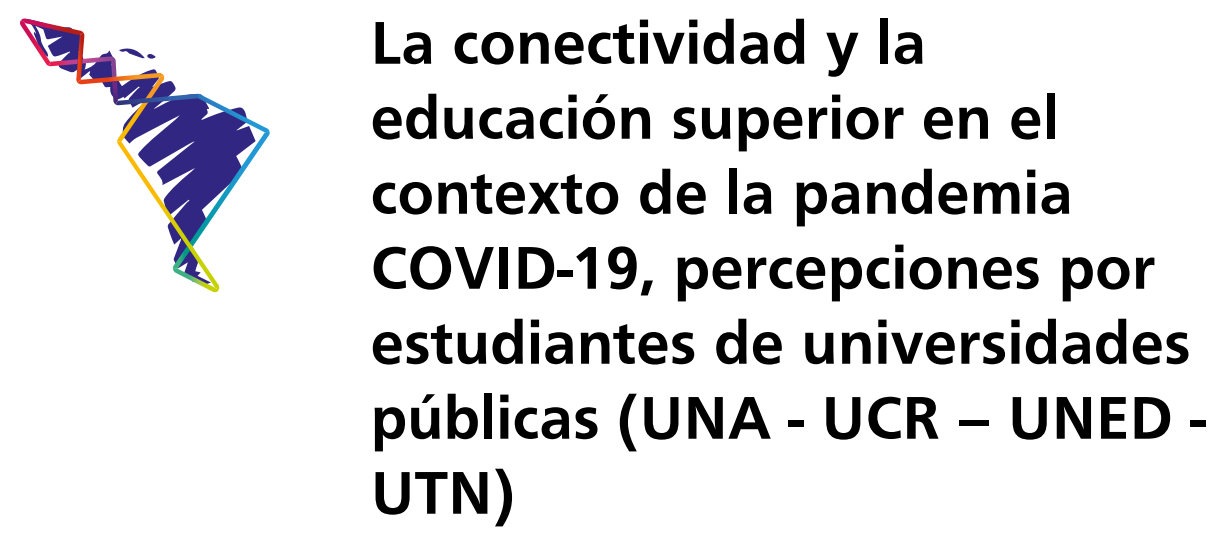

\title{
Connectivity and higher education
} in the context of the COVID-19 pandemic, perceptions by students of Public Universities (UNA - UCR - UNED - UTN)

\section{Conectividade e ensino superior no contexto da pandemia COVID-19, percepções de alunos de Universidades Públicas (UNA - UCR - UNED - UTN)}

Ph. D. Carlos Luis Chanto Espinoza ${ }^{1}$ MAP. Jorge Luis Loáiciga Gutiérrez²

\section{Recibido: 4-8-2021 •Aceptado: 5-10-2021}

1 Académico, investigador de la Universidad Nacional de Costa Rica. Ph. D en Proyectos de la Universidad Internacional Iberoamericana (UNINI) - México. M. Sc. Gerencia en Administración de Proyectos de Desarrollo del Instituto Centroamérica en Administración Pública (ICAP). Licenciado en Informática con énfasis en Sistemas de Información por la UNA. Correo electrónico: carlos.chanto.espinoza@una.cr (10 https://orcid. org/0000-0002-3420-7259

2 Académico, investigador de la Universidad Nacional de Costa Rica. Doctorando en Proyectos con Especialidad en Investigación por la Universidad Internacional Iberoamericana (UNINI-Puerto Rico). Máster en Administración de Proyectos por la Universidad para la Cooperación Internacional (UCI) - Costa Rica. Licenciado en Administración con Énfasis en Gestión Financiera por la UNA. Correo electrónico: jorge. loaiciga.gutierrez@una.ac.cr. ㄴ. https://orcid.org/0000-0002-0421-3906 


\title{
Resumen
}

Este artículo explora las percepciones sobre conectividad en la educación superior frente a la pandemia de la COVID-19, por estudiantes de cuatro centros de educación superior de Costa Rica: Universidad Nacional de Costa Rica (UNA), Universidad de Costa Rica (UCR), Universidad Estatal a Distancia (UNED) y Universidad Técnica Nacional (UTN). Se presenta los resultados de una encuesta realizada a una muestra de 302 estudiantes activos de las universidades en mención de la provincia de Guanacaste, mediante un enfoque cuantitativo, descriptivo, no experimental, utilizando el cuestionario y la revisión literaria para compilar la información. Es remarcable los resultados que afirman el impacto provocado y la dependencia por la tecnología para la práctica educativa en tiempos de coronavirus, donde una buena conectividad puede marcar la diferencia para un buen desempeño estudiantil. Finalmente, se da por un hecho que existe una baja conectividad en las zonas rurales de Guanacaste, que hay estudiantes sin un equipo tecnológico adecuado para desempeñarse en la presencialidad remota, sumado a un mal servicio de Internet por distintos proveedores y dependencia de las computadoras portátiles y del celular para las actividades sincrónicas y asincrónicas programadas.

Palabras clave: TIC, conectividad, brecha digital, innovación pedagógica, educación superior.

\begin{abstract}
This article explores the perceptions on connectivity in higher education in the context of the COVID-19 pandemic by students from four public universities in Costa Rica, Universidad Nacional (UNA), Universidad de Costa Rica (UCR), Universidad Estatal a Distancia (UNED) and Universidad Técnica Nacional (UTN). The results of a survey carried out on a sample of 302 active students of the aforementioned universities in the province of Guanacaste are presented through a quantitative, descriptive, non-experimental approach, using the questionnaire and literary review to compile the information. It is remarkable the results that affirm the impact caused and dependence on technology for educational practice in times of coronavirus where good connectivity can make a difference for good student performance. Finally, it is taken as fact the existence of low connectivity in the rural areas of Guanacaste, the existence of students without adequate technological equipment to perform in remote presence, added to the poor internet service by different providers, and the dependence on laptops and cell phones for programmed synchronous and asynchronous activities.
\end{abstract}

Keywords: ICT, connectivity, digital divide, pedagogical innovation, higher education.

\section{Resumo}

Este artigo explora as percepções sobre conectividade no ensino superior ante a pandemia COVID-19 por alunos de quatro universidades públicas da Costa Rica, Universidad Nacional da Costa Rica (UNA), Universidad de Costa Rica (UCR), Universidad Estatal a Distancia (UNED) e Universidad Técnica Nacional (UTN). Os resultados de um inquérito realizado a uma amostra de 302 alunos ativos das referidas universidades da província de Guanacaste são apresentados através de uma abordagem quantitativa, descritiva e não experimental, utilizando o questionário e a revisão literária para compilar a informação. É notável os resultados que afirmam o impacto 
causado e a dependência da tecnologia para a prática educacional em tempos de coronavírus onde uma boa conectividade pode fazer a diferença para o bom desempenho do aluno. Por fim, parte-se do pressuposto de que há baixa conectividade nas áreas rurais de Guanacaste, que há alunos sem equipamentos tecnológicos adequados para atuar em presença remota, somado ao precário serviço de internet por diferentes provedores e dependência de laptops e do celular para atividades síncronas e assíncronas programadas.

Palavras-chave: TIC, conectividade, exclusão digital, inovação pedagógica, ensino superior.

\section{Introducción}

Esta crisis mundial derivada por la enfermedad infecciosa nombrada como la COVID-19 ha provocado un replanteamiento de los servicios educativos a todos los niveles. La forma en que se imparte la educación, cambió en un cerrar de ojo, debido a la enfermedad del coronavirus.

Hoy, el salón de clases y el hogar, son el mismo lugar. Sobre esto, la Organización de las Naciones Unidas para la Educación, la Ciencia y la Cultura (UNESCO, 2020a), señala que al 31 de julio del 2021, existen 31453440 estudiantes afectados; de los cuales para Costa Rica se tiene un total de 1317482 educandos que se han visto enmarcados por las prohibiciones debido a la pandemia en Costa Rica. Tal y como lo establece la figural.

Figura 1. Impacto de la COVID-19 en la educación

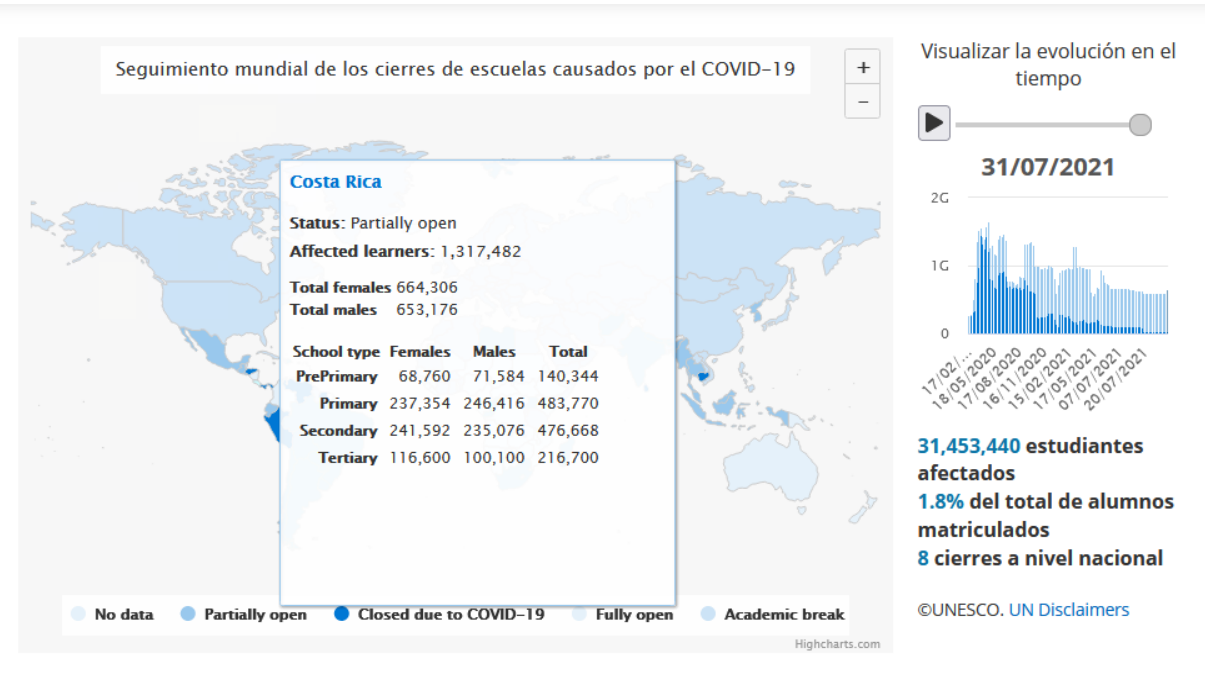

Nota: https://es.unesco.org/COVID19/educationresponse 
Todo un reto, ya que en muy poco tiempo se tuvo que reorganizar el sistema educativo, para que los estudiantes lograran continuar el proceso de enseñanza y aprendizaje a distancia, desde sus hogares. En el nivel de la educación superior la UNESCO (2020b), señala que "desde su fundación, las universidades, como cualquier otra institución social, han tenido que enfrentarse a epidemias devastadoras que han impactado en su funcionamiento cotidiano. Y han sobrevivido y continuado con su misión aun con las puertas cerradas." (p. 12)

Para Costa Rica, una de las medidas tomadas en materia educativa para afrontar la pandemia, a finales del mes de marzo de 2020; fue la de cerrar los centros de enseñanza (guarderías, escuelas, colegios), así como las instituciones de educación superior. Sin embargo, a raíz del confinamiento fue indiscutible el cambio acelerado en las metodologías de enseñanza y aprendizaje provocando un crecimiento exponencial de dependencia a las Tecnologías de la Información y la Comunicación e (TIC).

Asimismo, la UNESCO menciona "El uso intensivo de todo tipo de plataformas y recursos tecnológicos para garantizar la continuidad del aprendizaje es el experimento más audaz en materia de tecnología educativa, aunque inesperado y no planificado". (p. 6)

En este marco, la educación virtual pasó a adquirir una gran preeminencia para la sociedad en su todo, relevando brechas significativas con relación a la diversidad de habilidades y recursos disponibles por parte de la población estudiantil, tanto para acceder a este método de formación. Sin duda alguna estas discrepancias se han transfigurado en los mayores retos a solucionar por parte del sistema de educación superior del país.

En análisis al párrafo anterior, la pandemia en el contexto de la educación superior ensanchó las desigualdades en materia de las telecomunicaciones para la práctica educativa a distancia, en este sentido no todos los actores dentro del proceso pedagógico contaban con los requerimientos mínimos necesarios para desplegar fácilmente la virtualidad.

Así mismo evidencio una problemática de conectividad como factor determinante en la incursión de una nueva forma de hacer los procesos, donde la mediación pedagógica con atención formativa tradicional fue reemplazada por un modelo educativo denominado presencialidad remota con ayuda de herramientas tecnológicas.

Esta investigación busca conocer las percepciones acerca de la conectividad en la educación pública superior costarricense, considerando que a pesar de sus beneficios 
se presentan limitaciones que amplían las diferencias en la comunidad estudiantil en materia tecnológica. En la actualidad, educar sin conexión acrecienta las desigualdades y estas variables a favor de unos y en contra de otros se conoce como brecha digital, que aún representan un obstáculo cuando en materia de enseñanza se incorporan las TIC.

Con motivo a este factor tan determinante, "brecha digital", en la conectividad, cada centro de enseñanza de educación superior tuvo que ajustar con ayuda de las TIC, herramientas que permitieron a los estudiantes continuar con cada etapa de su aprendizaje, Trujillo, (2020a). Con la ayuda esta solución digital, y desde sus hogares cada alumno hace un esfuerzo para continuar su aprendizaje con teléfono celular y mecanismos como WhatsApp o correo electrónico. Vallespín, (2020); asimilando sobre el camino cómo manipular aplicaciones de trabajo en grupo online y videoconferencia; adaptándose a la estructura "de enseñanza" imputada por las plataformas tecnológicas. Trujillo, (2020b) y Brenner, (2020).

Realizar un cambio de una educación presencial a una modalidad virtual con ayuda de soluciones digitales es todo un reto, tal y como lo define Gonzales-Zamora et ál., (2020). De la misma forma Hodges et ál., (2020) y Álvarez, (2020a), mencionan que lograr llegar a todo el estudiantado de la noche a la mañana, sumado a las múltiples dificultades, tales como conectividad, falta de equipo en los hogares, formación en la utilización de las los recursos, aplicación de currículo en modalidad virtual, y otros, es sin duda alguna una barrera para eliminar en este nuevo siglo.

Por otra parte, acceder a la conectividad es una pieza fundamental para la nueva era del sector educación, es un recurso que ha venido a modificar la práctica educativa sobre todo en el nivel universitario, pensar en un mañana sin Internet no es factible, considerando que estamos ante una industria 4.0. que demanda generaciones con competencias en lo digital.

Esta investigación permitirá al lector visualizar las percepciones que han vivido los educandos de las cuatro universidades públicas del cantón central de Liberia, Guanacaste: UNA, UCR, UTN y UNED en materia de conectividad en tiempos pandémicos, permitiendo asumir un criterio propio de los pros y contra que viven las personas que se muestran vulnerables y frágiles ante una situación de inequidad. Existe todavía una enorme brecha digital dentro de nuestro país y entre cada región provincial y cantonal. 


\section{Objetivo general}

El objetivo de esta investigación consiste en describir las percepciones sobre conectividad en la educación superior, frente a la pandemia de la COVID-19, por estudiantes de cuatro universidades públicas de Costa Rica: Universidad Nacional de Costa Rica (UNA), Universidad de Costa Rica (UCR), Universidad Estatal a Distancia (UNED) y Universidad Técnica Nacional (UTN) del Cantón de Liberia, Guanacaste, Costa Rica.

\section{Fundamentación teórica}

La pandemia provocada por la COVID-19 se ha convertido en uno de los desafíos más magnos que ha tenido que afrontar la sociedad mundial durante el presente siglo. Con el propósito de frenar la transmisión de esta enfermedad, Costa Rica ha transformado el distanciamiento físico en una opción no farmacéutica de gran masividad, igualmente ha causado que los sistemas de Educación Superior interrumpan la formación presencial tradicional, creando medidas de atenuación de impacto que concuerden con la realidad nacional.

Para lograr este proceso de cambio el componente determinante es la conectividad, que mediante el uso de Internet en establecimientos educativos ha facilitado la pedagogía del profesorado y del estudiantado y de todos los actores que forman parte del sector formativo superior, sin embargo, se presenta un fenómeno llamado brecha digital que ha sido un reto para el sector. En esta perspectiva, Martínez (2020) menciona:

(...) La brecha digital es, ante todo, expresión de la desigualdad en el uso y acceso de las nuevas Tecnologías de la Información y la Comunicación (TIC), y como desigualdad es generadora de procesos de exclusión social. Las causas que acompañan a su aparición son múltiples, desde la falta de las competencias y destrezas necesarias para el uso de las TIC, hasta la imposibilidad de acceder a los recursos electrónicos y de conexión necesarios, debido a bajos umbrales de renta. (p. 267).

Mientras tanto, Anaya et ál., (2021) indica que "las brechas digitales se acentúan por factores de conectividad, dispositivos, apropiación de la tecnología, inadecuada formación docente, entre otros" (p. 12). Entre tanto, conviene subrayar lo que afirma Vivanco-Saraguro (2020); “(...) los hogares del estrato socioeconómico más desfavorecido, aquellos de los grupos tradicionalmente excluidos y marginados están en obvia desventaja al no contar con acceso a internet, lo cual les impide continuar con el proceso educativo de sus hijas/os" (p. 7). 
Recíprocamente López (2020) manifiesta que la "brecha digital que se traduce en desigualdad educativa y social. En un contexto de vulnerabilidad, las familias no disponen de los recursos electrónicos suficientes ni de un acceso óptimo a internet" (p. 268). Así mismo, cada estudiante se ve envuelto en un nuevo contexto de ambientes familiares, apoyándolos cuando podían en cada actividad. Tal y como lo establece Mian, A., y Khan, S. (2020).

Estamos siendo testigos de situaciones en donde la brecha digital fue expuesta: en la cual la comunidad universitaria se veía saturado de "deberes" y múltiples actividades académicas, tal y como lo definen la Confederación Española de Asociaciones de Madres y Padres del Alumnado (CEAPA), (2020), forzados a permanecer en casa y continuar las clases en modalidad digital durante un extenso periodo de aislamiento, según lo mencionan autores como Wang, Zhang et ál., (2020).

Por otra parte, Gonzales-Zamora et ál., (2020) señalan:

(...) en consecuencia, esta crisis ha provocado que millones de estudiantes, de todos los niveles educativos, se hayan visto obligados a seguir el curso académico sin docencia presencial, escenario que plantea muchas dudas: ¿están los diferentes colectivos preparados para asumir el reto?, ¿cuentan los estudiantes con todos los recursos necesarios para continuar el curso a distancia?, ¿está el sistema educativo preparado para atender a las necesidades y diferencias individuales para llegar a todos?. (p. 104)

Muchos estudiantes tuvieron que equiparse a veces con dispositivo celular con escasa señal y almacenamiento, para ingresar a aquellas plataformas con las que los centros universitarios y el profesorado disponían las actividades. De la misma forma, se vuelve todo un desafío poder participar en videoconferencias o video llamadas colectivas, por la muy poca o nula señal.

Sumado a esto, el estar en casa con familiares que se acaban de quedar sin empleo, y que tienen que continuar pagando los alimentos y los servicios comunes. En su defecto, plantea Torices, (2020), “(...) alumnos y alumnas que tienen dificultades para acceder a internet porque no tienen ordenadores o porque viven en zonas a las que la señal no llega con la suficiente intensidad o porque tienen problemas para compartir la banda ancha".

La pandemia provocada por la COVID-19 ha causado una de las mayores paralizaciones del proceso enseñanza aprendizaje en la historia de Costa Rica, similar a diversos países, esta nación presentó la necesidad de cerrar los centros educativos y emigrar a métodos de enseñanza a distancia y mixta, sin que las instituciones de 
estudio, ni las células familiares truncaran con la capacidad pedagógicas, ni las herramientas digitales obligatorias para reconocer los desafíos de la educación virtual.

Por ende, una importante cantidad de estudiantes en el nivel nacional no lograron prolongar las clases de forma remota, debido a la falta de conectividad en su zona geográfica, de equipo tecnológico. En relación a la idea anterior el sitio del Fondo de las Naciones Unidas para la Infancia Unicef (2021), establece:

En el mundo 3.700 millones de personas no pueden acceder a Internet. Globalmente, 2.200 millones de niñas, niños y jóvenes menores de 25 años (dos tercios de las niñas, niños y jóvenes de todo el mundo) no tienen conexión a Internet en casa. En América Latina y el Caribe esta cifra alcanza los 130 millones de personas, la mayoría pertenecen a comunidades y grupos altamente vulnerables. (párr. 4)

En el contexto de la investigación, establecida en una muestra de estudiantes universitarios de comunidades rurales, no todos los educandos disponen de un recurso tecnológico (computadora), mucho menos de una adecuada cobertura de banda ancha que proporcione el acceso expedito y constante a la información.

Esta indiscutible brecha digital, demarca un obstáculo para poder recibir una educación de calidad por parte del estudiante, a través herramientas tecnológicas, ya sean plataformas educativas o aplicaciones (apps) como WhatsApp.

Lo mismo ocurre con Wanderley y Calvo (2020), quienes mencionan:

(...) Otro elemento importante, en caso de que se tenga el acceso a internet y la disponibilidad de los aparatos (computadora, Tablet, etc.) es el acceso a las plataformas. La gran variedad de plataformas ofertadas (Blackboard, Google Classroom, Moodle, Seesaw) y las opciones para mantener reuniones y clases virtuales (Zoom, Meets, Teams) hacen que sea imprescindible evaluar la calidad, pertinencia, utilidad de éstas para nuestra población estudiantil y sus diferentes necesidades. También se debe evaluar la posibilidad de que los maestros y estudiantes accedan a éstas por el costo y los requerimientos tecnológicos que muchas tienen (p. 8).

Contrariamente de los esfuerzos del gobierno que, desde inicios de la pandemia, ha sido de su conocimiento que para las familias de más escasos recursos y vulnerables, aprender desde el hogar puede ser un reto insuperable, esto debido a la ausencia de equipo tecnológico, por la falta de acceso a Internet, o incluso por carecer de un sitio en su hogar, donde sentarse a estudiar.

Los atrasos en la educación se vienen recolectando desde el 2018, con la histórica huelga de cuatro meses, de los docentes; igual en el 2019, se perdió al menos un 
1 mes de clases efectivas mientras que, en el 2020, la pandemia provocada por la COVID-19, propicio desafíos en el proceso de enseñanza aprendizaje, estableciendo demoras en quienes no contaban con los recursos. Sin duda alguna la brecha se ha dilatado entre los estudiantes que han tenido ayudas, así como acceso a una adecuada conectividad y quiénes no tienen esas ventajas. Según lo establece el Programa Estado de la Nación (2020) “(...) debido a la brecha digital, que se manifiesta en un acceso parcial a conexiones de Internet estables, pues no todas las viviendas ni en todas las zonas del país esta ha sido una opción viable para muchos estudiantes..." (p. 68).

A raíz de este fenómeno cada institución de educación superior impulsó plataformas que intentaban dar soporte (Zoom, Microsoft Team, Google Hangouts, entre otras), y espacios web (aulas virtuales), donde incorporaban recursos educativos antes diseminados, para docentes, estudiantes y familias, como ya se había experimentado en otros países, tal y como lo menciona Zhang et ál., (2020) y Wang, Cheng, et ál., (2020).

Autores como Gewin, (2020) y Boursicot et ál., (2020), hacen mención que, ante el reto de la virtualidad, se establecieron metodologías de enseñanza y evaluación online que se habilitaban sobre la marcha, como en múltiples partes del mundo que vivían el mismo contexto.

Es importante mencionar que cada organización en el nivel de educación superior, realizó múltiples estrategias, como lo fueron entrega de tarjetas chip con datos para celulares; tablet y portátiles, entre muchos estudiantes sin herramientas tecnológicas, tal y como lo menciona Torices, (2020).

En este marco, la educación virtual pasó a adquirir una gran preeminencia para la sociedad en su todo, relevando brechas significativas con relación a la diversidad de habilidades y recursos disponibles por parte población estudiantil, tanto para acceder a este método de formación. Sin duda alguna estas discrepancias se han transfigurado en los mayores retos a solucionar por parte del sistema de educación superior del país conociendo de antemano que hay mucho camino por recorrer en materia de conectividad.

\section{Metodología}

Se empleó una investigación desde el enfoque cuantitativo con una profundidad de tipo descriptiva bajo un estudio no experimental de alcance longitudinal, considerando que los datos fueron recopilados en distintos momentos en el tiempo (meses de junio y julio de 2021). Utilizando la estadística descriptica con frecuencias relativas y absolutas a lo largo de la investigación. 
Las técnicas para la recolección de datos fueron la revisión de la literatura y la encuesta, siendo esta última la que mayormente considera los componentes descriptivos en el estudio. Ahora bien, con objeto de esta investigación se creó e implementó un cuestionario online remitido a estudiantes de cuatro universidades públicas: UCR, UNA, UNED y UTN ubicadas en el cantón de Liberia, Guanacaste, Costa Rica. Por consiguiente, la muestra se configuró mediante la plataforma tecnológica Google form, lo que posibilitó un alcance extendido y comparativo en los resultados.

\section{Muestra y procedimiento}

En el estudio participaron 302 estudiantes de las diferentes universidades públicas antes mencionadas, bajo un muestreo no probabilístico dentro de un período donde las clases se desarrollaron desde la modalidad de presencialidad remota. Se empleó un cuestionario estructurado y cerrado de escasas preguntas, enviado por correos electrónicos institucionales y por la vía telefónica mediante la aplicación digital WhatsApp. De esta muestra el 65,9\% eran mujeres y el 34,1 \% hombres con edades comprendidas entre los 17 y 25 años.

\section{Resultados}

El análisis descriptivo de los datos permite conocer las características generales que definen a las personas estudiantes que participaron de la investigación. De acuerdo a la información suministrada y reflejada en la figura 2 existe una tendencia mayoritaria de colaboración en el estudio por parte de los aprendientes de la UNA con un $43,7 \%$, en cuanto a las demás universidades: la UTN un $22,5 \%$, la UNED con $19,2 \%$ y finalmente la UCR cerca del $14,6 \%$ del estudiantado 
Figura 2. Población participante de la investigación

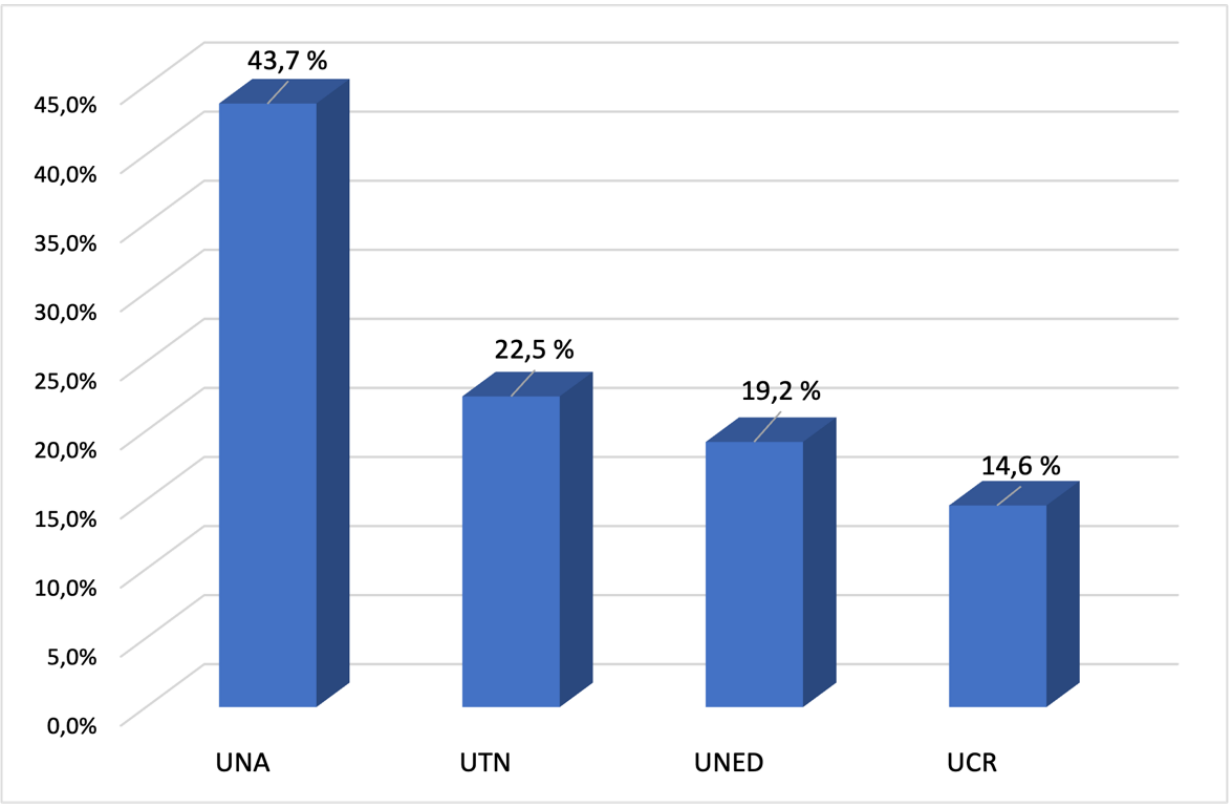

Nota: elaboración propia (2021)

Por otra parte, para desplegar con éxito la modalidad educativa denominada presencialidad remota, es fundamental que los jóvenes universitarios presenten una buena conectividad en materia de Internet, así como equipos electrónicos con todos los requerimientos adecuados. Es por este motivo que se les preguntó algunos aspectos relacionados con conectividad.

En primera instancia se evaluó la conexión actual a Internet entre los participantes donde el 42,7\% (132 en términos absolutos) indica un mal servicio de internet, un $41,7 \%$ (126 estudiantes) empresa regularidad y únicamente el 15,6\% (47 encuestados) un buena conectividad. En esta perspectiva los aprendientes de las diferentes universidades públicas manifestaron que para realizar sus actividades académicas en línea todos en un $100 \%$ lo hacen desde entornos virtuales de aprendizaje desde la plataforma de Moodle.

Ahora bien los resultados muestran que alrededor del 70,5\% se conecta al web educativa desde su casa de habitación, el 36,8 \% desde los datos de su celular personal postpago que sin duda es una cantidad importante, un 20,9\% utiliza móviles prepagos, unos pocos, $1,7 \%$ buscan lugares públicos como parques, centros comerciales, bibliotecas y lo más crítico del análisis el 2,3\% indicó no tener Internet, 
a raíz de este problema no solo usan las áreas públicas antes mencionadas, sino que también su lugar de trabajo, casa de conocidos, vecinos que prestan la señal inalámbrica o incluso se reúnen con sus compañeros de clases para conectarse.

Otro aspecto importante de analizar son los dispositivos tecnológicos que utilizan con mayor frecuencia los estudiantes encuestados para realizar sus distintas actividades desde la presencialidad remota, así lo manifestó mayoritariamente el 78,8\% que indicó el uso de la computadora portátil. Además, un 76,8 \% señalo disponer de un teléfono celular con acceso a Internet. Ver figura 3 para un mayor detalle:

Figura 3. Herramientas tecnológicas utilizadas por los encuestados para la práctica educativa remota

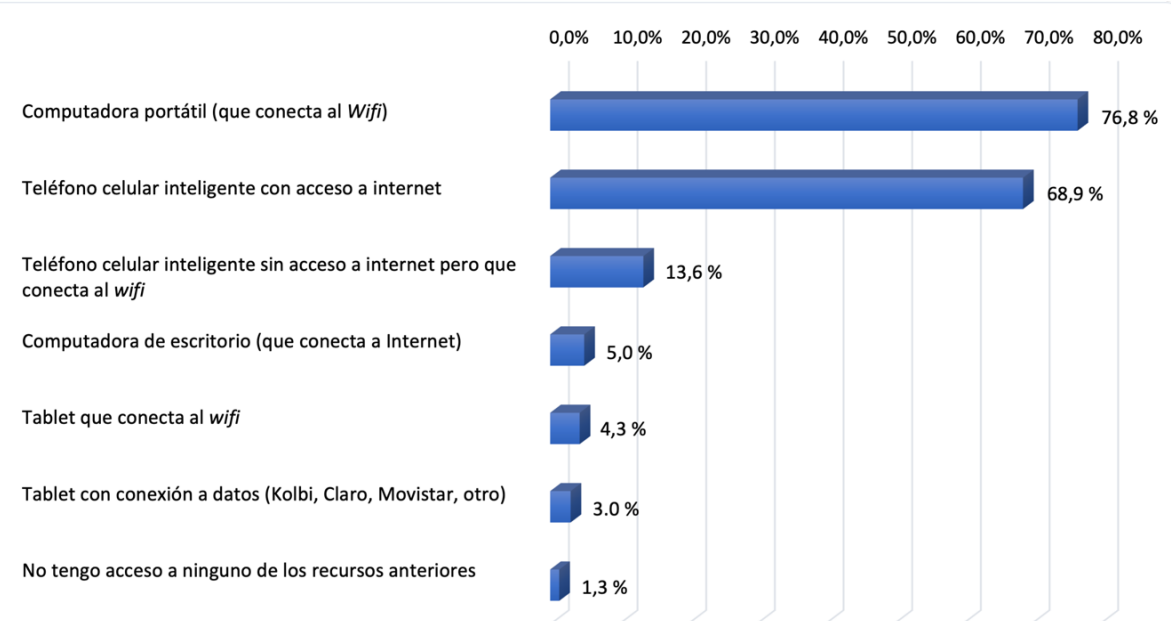

Nota: Los porcentajes no suman 100 porque los participantes podían seleccionar más de una opción. Elaboración propia (2021)

La figura 4 muestra las herramientas tecnológicas que mayormente utilizan los sujetos encuestados para realizar sus actividades universitarias desde lo asincrónico y lo sincrónico, donde las de mayor uso son el ZOOM, el aula virtual institucional (Moodle) y el WhatsApp con 69,2 \%, 65,6 \% y 63,6 \% respectivamente.

La existencia de estas herramientas de apoyo resulta fundamental en el logro de la práctica educativa sobre todo para enfrentar el confinamiento generado por la pandemia de la COVID-19, cuyos efectos trascienden no solo lo educativo, sino lo social, económico, cultural, político y ambiental del país. Asimismo, los estudiantes han promovido la diversidad de plataformas digitales como, por ejemplo: Google 
drive, Meet, Classroom, Youtube, One drive, Skype, Microsoft Teams, Facebook, Telegram, Dropbox, y correos electrónicos, entre otros.

Figura 4. Herramientas tecnológicas utilizadas por los encuestados para la práctica educativa remota.

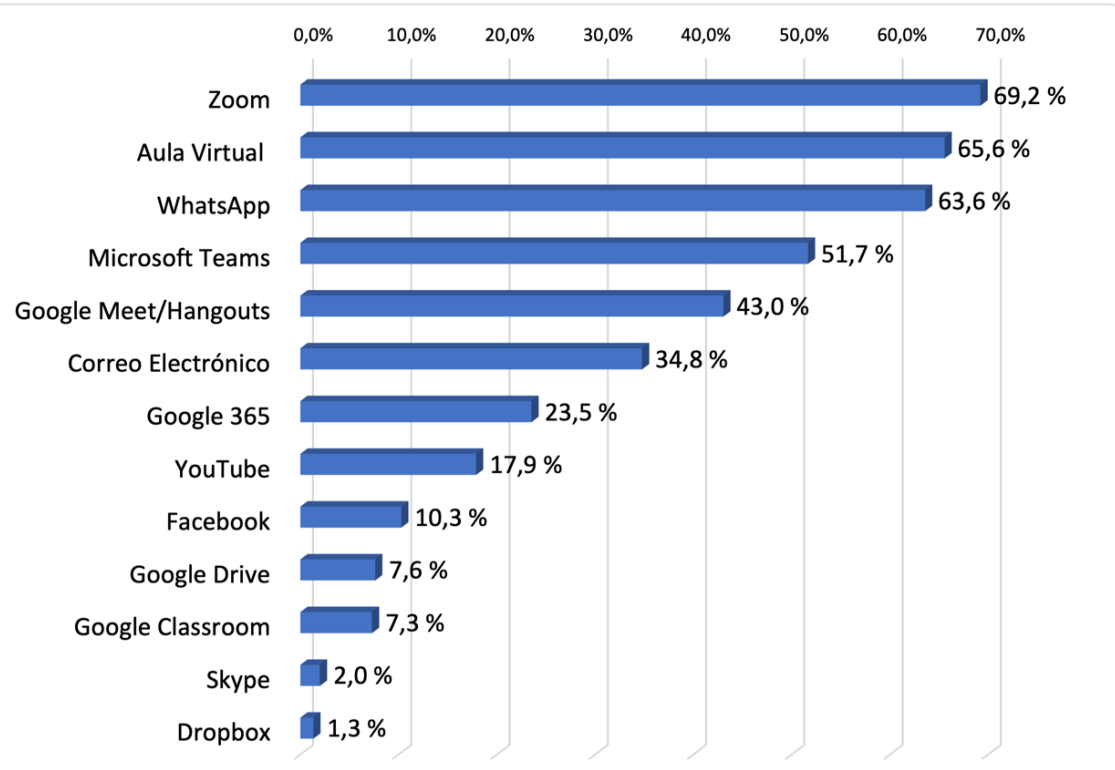

Nota: los porcentajes no suman 100, porque los encuestados podían seleccionar más de una opción. Elaboración propia (2021)

Para continuar se les consulto a los encuestados el nivel de satisfacción que podrían brindar de la conectividad, para la práctica educativa en tiempos de la COVID-19, todo con base en la zona geográfica donde viven actualmente. El resultado se dio en una escala Likert (5 ítems) en donde su mayoría 48,3 \% (146 valor absoluto) indicó ser buena, pero apenas un 2,6\% ser excelente, por lo que de manera general hay mucho que mejorar en términos de internet sobre todo en regiones rurales de la provincia de Guanacaste.

En esta perspectiva general el $36,8 \%$ manifiesta tener como proveedor del ciberespacio la compañía Kolbi, el 21,2 \% Cable Tica, 18,5 \% Tigo y con 18,2 \% y 17,2 $\%$ Claro y Movistar, respectivamente. La preferencia se inclina sobre el Instituto Costarricense de Electricidad (ICE), quienes poseen los derechos sobre compañía telefónica antes mencionada. 
En este orden de ideas los resultados indican que entre los inconvenientes más reiterativos que se han presentado en el nivel de conectividad, es decir de la red de comunicación que permite la interconexión entre computadoras, tablets, celulares inteligentes, las portátiles, entre otros están:

Figura 5. Problemas de conectividad durante la práctica educativa en presencialidad remota

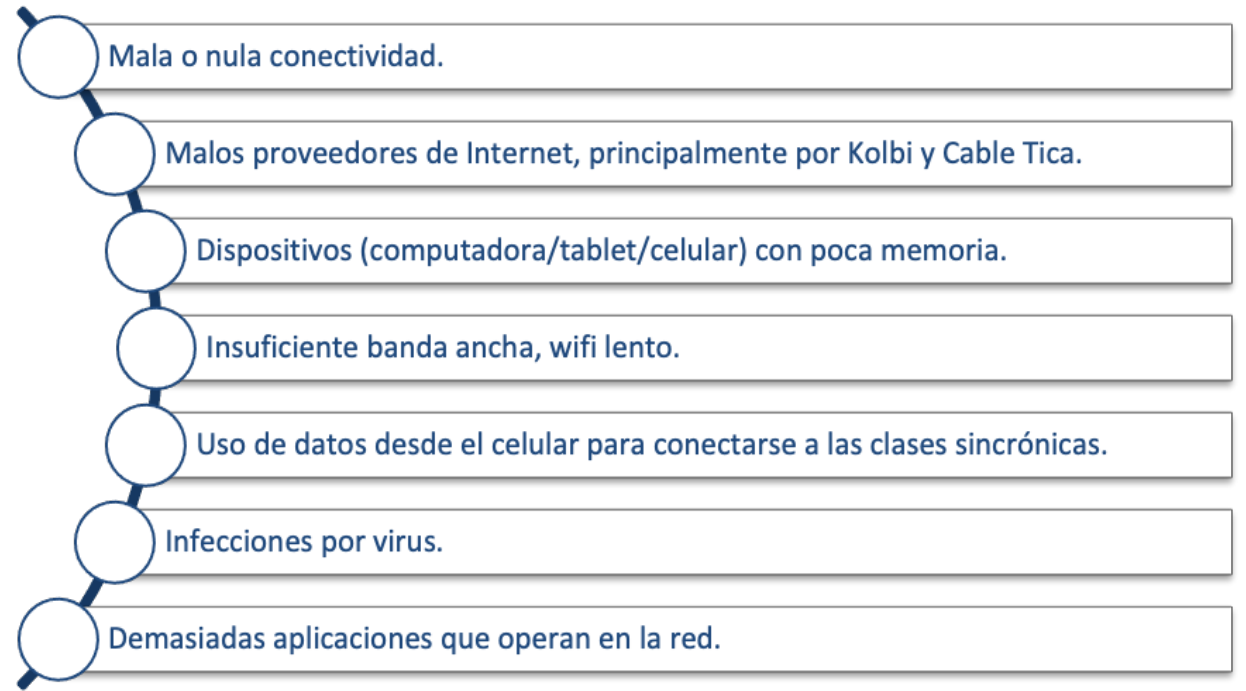

Nota: elaboración propia (2021)

Algunos otros inconvenientes que han experimentado los estudiantes frente a cambios en su práctica educativa, en tiempos de la COVID-19 y a nivel de la cotidianidad, producto del confinamiento y que tienen un impacto en la calidad de vida y la evolución social, se muestran en la figura 6: 
Figura 6. Inconvenientes por los encuestados frente a la práctica educativa en presencialidad remota

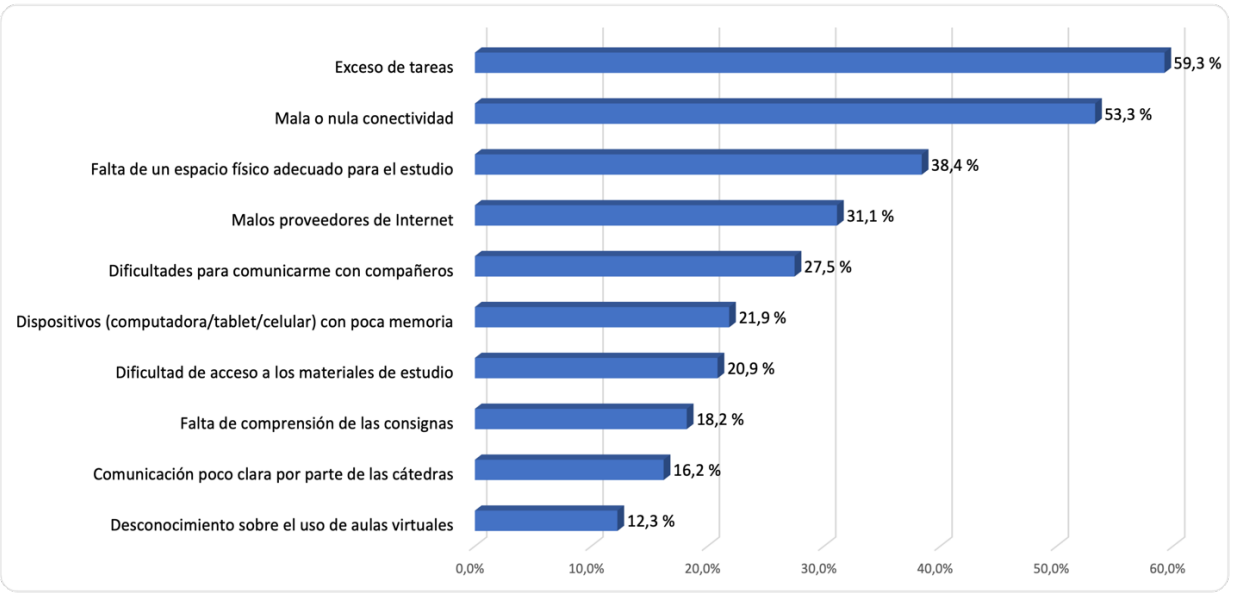

Nota: los porcentajes no suman 100 porque los encuestados podían seleccionar más de una opción. Elaboración propia (2021)

Retomando lo indicado en las figuras 5 y 6 los sujetos encuestados consideran que las universidades públicas deben mejorar el apoyo a la persona estudiante en el conocimiento de la tecnología a utilizar y mejorar la comprensión de la persona docente en el uso de las herramientas digitales que emplea, con consejos como:

Mantener actividades sincrónicas cortas (videoconferencias), no exceder las asincrónicas durante la semana, tener una real comprensión de los problemas de Internet que presenta el estudiantado (empatía), brindar ayudas con equipo tecnológico, mejorar la comunicación profesor-estudiantes, entre otros.

Por otra parte, la pandemia obligó a tener que implementar múltiples cambios en la metodología de enseñanza y aprendizaje adoptando nuevos patrones en el quehacer académico al pasar de la presencialidad a una modalidad remota. Esta situación dio origen a la necesidad de conocer la percepción que tenía el estudiantado de su experiencia para conectarse a las clases y del desarrollo de sus actividades online durante el I ciclo 2021.

En términos generales a continuación se presentan extractos de las principales percepciones de la población estudiantil de las cuatro universidades públicas en estudio donde se muestran las experiencias vividas durante el quehacer diario de actividades sincrónicas y asincrónicas desde la presencialidad remota durante el I ciclo de 2021 : 


\section{Tabla 1}

Extractó de las percepciones de conectividad en los participantes de la UNA

- "Que muchas veces el acceso al Internet es ilimitado". "Ha sido un cambio drástico, pues se debe de acostumbrar a limitar el tiempo de trabajo y distribuirlo entre el hogar y lo académico y otros".

- "Nada satisfactorio, ha sido muy difícil todo el proceso".

- "Mucho estrés y problemas psicológicos".

- "Hay menos comunicación entre docente y alumno Mayor frustración, invasión de los lugares de descanso (cuarto de la casa se convirtió en el aula)".

- "Los profesores se exageran con el tiempo de clases. Eso es un mal sabor, porque no consideran las situaciones que uno vive en cuanto a salud y economía".

- "Durante la pandemia ha sido la mejor forma para seguir con los es-

UNA tudios, sin embargo, para muchos se ha hecho difícil, ya que como lo es mi caso, vivo en una zona sin Internet y los estudiantes debemos de buscar la manera de cómo estar al tanto de nuestras actividades académicas".

- "A mí me ha costado aprender el uso de la tecnología y en mi comunidad no hay casi señal para el celular".

- "Es muy estresante, a veces muy poco claro, pero a la vez es una gran oportunidad para continuar y no atrasarse".

- "Principalmente me ha dejado niveles de estrés muy altos, y que el uso de la tecnología es mucho más útil de lo que se cree".

- Sin dudas fue grande el cambio, en aprender a utilizar herramientas tecnológicas que antes no conocía o utilizaba".

- "La conectividad ha sido malísima".

Nota: elaboración propia (2021) 


\section{Tabla 2}

Extractó de las percepciones de conectividad en los participantes de la UTN

- "Es muy complicado, porque el Internet es muy inestable y es dificil poner el $100 \%$ de atención a la clase por interferencias".

- "Falta de mejor comprensión de los contenidos, debido a las limitaciones en cuanto a la mala conexión a Internet y acercamiento a los profesores y de equipo adecuado."

- "Una percepción desalentadora, porque no es lo mismo, casi no se aprende y son demasiados trabajos y tareas por semana, además

UTN son muchas horas frente a la computadora."

- "Recibir clases de manera virtual es más difícil, estresante, cansado y desesperante de lo que pensaba."

- "Conocer aplicaciones que no sabía que existía para podernos comunicarnos con personas dentro y fuera del país."

- "Fue un cambio radical, aunque ya me he adecuado, aun así, prefiero la presencialidad para mis estudios profesionales." 


\section{Tabla 3}

Extractó de las percepciones de conectividad en los participantes de la UNED

- "La flexibilidad y adaptación que debemos tener ante este tipo de cambios, además de que la virtualidad nos permite tener horarios más flexibles con el estudio".

- "Incertidumbre y confusión, el estrés es muy elevado".

- "La clave es mejorar el autoaprendizaje y la misma aún no se desarrolla.

- "Siento que existe desigualdades en materia de conectividad".

UNED

- "Buena por una parte porque se han conocido herramientas tecnológicas que han resultado ser útiles en la actualidad y mala por otro lado porque por cuestiones de señal y conectividad que poseo, en muchas ocasiones no logro participar como se debe en las clases virtuales".

- "En lo personal me ha dejado desanimado".

- "Hay mucha distracción, así como problemas de conexión que dificulta recibir clases".

- "Por el Internet es más complicado si fuera bueno mi Internet no tendría queja".

Nota: elaboración propia (2021) 


\section{Tabla 4}

Extractó de las percepciones de conectividad en los participantes de la UCR

- "Es muy complicado para muchas personas con pocos recursos económicos, que no podemos contratar un buen servicio de Internet".

- "Ha sido un gran cambio, ya que al pasar del colegio a la universidad es un cambio muy grande, ya que se exige el triple y la tecnología es superior. Pero he aprendido grandes cosas que me ayudarán en el transcurso del año".

- "No existe igualdad de condiciones en cuanto la conectividad".

- "El pasar de una modalidad presencial a virtual, ha sido más difícil, ya que la mayoría tenemos el doble de estrés que en las clases

UCR presenciales".

- "El cambio al inicio fue complicado por la inactividad que teníamos estudiantes y profesores en las plataformas virtuales, con el tiempo se han desarrollado habilidades en el uso de las mismas y ha facilitado el proceso de aprendizaje, permitiendo que el estudiante pueda soportar más la carga académica. Por otra parte, sería bueno que los docentes no hicieran tan extensas las clases sincrónicas y que no se sobrepasen con tantos trabajos extraclases".

- "Siempre un cambio es difícil y se vuelva más difícil con las malas conectividades, por el motivo de que las líneas solo son buenas en la parte urbana y no en la rural".

Nota: Elaboración propia (2021)

\section{Sistematización de los hallazgos encontrados en las percepciones de conectividad}

Como resultado de las percepciones indicadas en las tablas 1, 2, 3 y 4 respectivamente, en materia de conectividad por los participantes del estudio en la figura 6 se desprenden los siguientes hallazgos sistematizados a través de un cubo que resume los 4 principales problemas encontrados: 
Figura 7. Problemas de conectividad durante la práctica educativa en presencialidad remota.

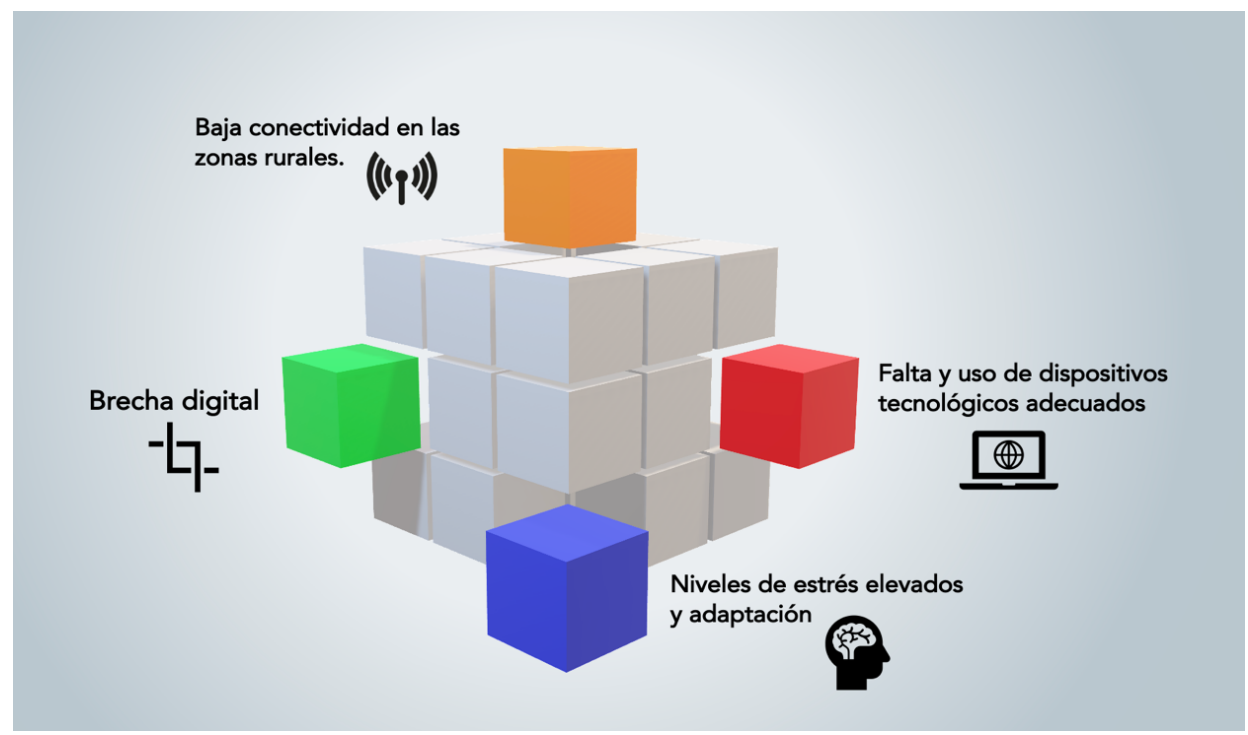

Nota: elaboración propia (2021)

Todos los puntos que sobresalen en la figura 6 nos describen un panorama no alentador porque a pesar de los grandes beneficios que trae consigo la conectividad, los aprendientes han sido sometidos a la utilización de herramientas tecnológicas de forma abrupta, como estrategia tomada por las autoridades de educación superior, en donde el estudiantado ha tenido que realizar grandes esfuerzos para adaptarse a la nueva modalidad.

Esto a pesar de factores intrínsecos como: fallas de conexión o falta de tecnología por no contar con los recursos económicos, la poca socialización y exceso de cargas de trabajo que conlleva a altos niveles de estrés, distracciones comunes del hogar, cansancio y dificultades que se manifiestan aún más, tras la problemática de la brecha digital.

Los hallazgos finales sumados al problema de investigación y el objetivo propuesto, desprenden por parte de los participantes una exigua complacencia por dificultades de conectividad, ausencia de capacidades para la educación virtual, combinación de distractores, así como la mención de menor calidad del aprendizaje.

Por otra parte, la población estudiantil que forma parte de esta investigación, además de los cuatro componentes especificados en el la figura 7, también, evidencian poca 
satisfacción, ya que la experiencia del proceso de enseñanza aprendizaje durante el aislamiento académico especifica copiosas dificultades, tales como: disminuida motivación y un sentimiento de soledad.

Sumando a esto, restricciones para distintas actividades educativas como, por ejemplo: realizar prácticas supervisadas, falta de tiempo, por la gran cantidad de tareas, ausencia de capacidades para desempeñarse en métodos formativos online, deducción de la calidad del aprendizaje y abundancia de distractores durante los encuentros sincrónicos.

Admitir que los equipos tecnológicos y el acceso a la conectividad no son elementos externos, sino una forma complementaria en nuestras vidas, deslumbrando así la premura de políticas institucionales y públicas que conciban la conectividad y estas tecnologías como un derecho. La pandemia desnudó las prácticas del proceso enseñanza aprendizaje emergentes que exponen transformaciones didácticas significativas, atrayentes, y pujantes.

Nos congregó a cuestionar los procedimientos curriculares; y resulta indispensable reconocer que, si no existe una red de comunicación de alcance global (Internet), la educación se transforma en un privilegio. Por ende, es puntual situar en el breviario político la imagen de que la conectividad es un derecho humano, que consiente el acceso a otros elementos como el de una educación básica, pública, gratuita, y universal.

\section{Conclusiones}

Los resultados muestran, que los hogares de los estudiantes encuestados pertenecientes al sector rural cuentan con una velocidad de conexión baja que limita o no permite la educación remota de calidad y que muchos dependen de sus datos celulares, aun teniendo computadoras portátiles, para recibir sus clases y desempeñarse en actividades asincrónicas. Sobre esto estamos de acuerdo con Wendelboe et ál., (2020) quien manifiesta:

Las brechas digitales entre estudiantes de escuelas urbanas y rurales están asociadas a diversos factores que las acentúan: la baja velocidad de conexión y la limitada presencia de red fija en las áreas rurales; el uso predominante por parte los estudiantes de dispositivos móviles no adecuados bajo la modalidad de prepago, con modelos de poca capacidad; la reducción de la apropiación de la tecnología por parte de los estudiantes de las áreas urbanas desde la temprana de edad y también de muchos profesores, evidenciándose que existen carencias de computadoras, no 
solamente por parte de los estudiantes, sino también de los profesores de las áreas rurales; elevados costos de los servicios fijo o móvil de internet (p. 29).

Ahora bien, nos queda claro que la realidad de la brecha digital, se deslumbró, gracias a la pandemia provocada por la COVID-19. El cierre temporal, de los centros de educación superior, estimulo verdaderas discrepancias entre contextos dentro de una misma zona geográfica, y las derivaciones, de nuevo, son característicamente graves para la persona estudiante en situación desfavorecida y con acceso muy restringido a las oportunidades educativas fuera de la presencialidad en el contorno universitario.

Asimismo, este contexto revela la dificultad que ha representado el cambio de la práctica educativa para todos, sin dejar de lado que la brecha digital ya de por sí abierta pueda ampliarse aún más y dejar ver sesgos y estratos sociales en el sistema educativo costarricense. Sobre esto Chanto y Loáiciga (2020) afirman:

Dentro de este marco, el acceso a las tecnologías digitales y el uso de la Internet en el territorio nacional ha sido todo un reto. Los efectos contradictorios del distanciamiento físico que está presenciando la población pueden ser compensados por una alta conectividad remota por medio de las tecnologías de información y comunicación (TIC). No obstante, esto no es opción para muchas familias costarricenses (p. 14).

Las universidades públicas deben de adquirir un papel protagónico, ejecutando nuevos e inminentes esfuerzos para progresar en el cierre de la brecha digital y certificar una enseñanza de calidad que reconozca las nuevas habilidades y necesidades para la vida y la empleabilidad que requieren las sociedades presentes.

Contar con una población con destrezas cognitivas y digitales apropiadas es un dominante para disputar y progresar en la economía mundial. El país sin duda alguna, ha avanzado buscando el desarrollo de una nación que garantice el derecho universal a su población, así como el manejo de las tecnologías de la información y la comunicación; tal y como se establece en el Plan Nacional de Desarrollo de las Telecomunicaciones (PNDT) 2015-2021 denominado Costa Rica: Una Sociedad Conectada.

En el presente trabajo se concluye que la mayoría de los estudiantes encuestados disponen de tecnología en su hogar, pero no poseen los recursos necesarios y los equipos con que cuentan no reúnen las condiciones mínimas para un buen desempeño en la práctica educativa. Además, se ha constatado que existe una carencia de habilidades blandas para asimilar el estrés causado por el confinamiento de la COVID-19. 
Por otra parte, los estudiantes de las universidades en estudio, UNA, UCR, UNED y UTN presentan similitudes en cuanto a brechas digitales, uso de herramientas tecnológicas, carencias en metodologías de aprendizaje y los inconvenientes que con anterioridad se han venido describiendo.

Finalmente, ante un contexto desafiante como el presente, se ha puesto en evidencia, la insuficiencia del sistema educativo universitario, donde este debería ser más flexible, y establecer una creciente capacidad para trabajar con apoyo de herramientas tecnológicas. En el mismo contexto se debe afrontar a fondo las medidas necesarias para certificar la igualdad de oportunidades a todos educandos.

Este trabajo de investigación pone en manifiesto que los recursos tecnológicos que dispone el estudiantado en el hogar, siendo estos limitados, repercuten en la continuidad de los estudios superiores. Se ha evidenciado que factores de conectividad, confinamiento, habilidades duras y blandas, y equipo tecnológico han incrementado la brecha digital. A raíz de esto se proponen las siguientes estrategias para la mejora del aprendizaje desde la presencialidad remota durante el brote de la COVID-19.

- Garantizar la equidad digital, a través de un plan estratégico universitario de conectividad que brinde apoyo con recursos tecnológicos al estudiantado (chip, tablet, portátiles, entre otros).

- Capacitar en el buen uso de las tecnologías de aprendizaje en línea al profesorado y el estudiantado desde los diferentes roles que representan en la práctica educativa.

- Realizar convenios con distintos proveedores de Internet, para promover mejoras del servicio, señal, costos, datos, entre otros.

- Valorar la posibilidad de que los estudiantes cuenten con la oportunidad de usar los servicios de los diferentes campus de estudio, grupos reducidos, en distintos horarios y con todas las medidas de salud.

- Cambiar las metodologías de enseñanza y aprendizaje desde el home office.

- Finalmente, descongestionar la red durante las actividades sincrónicas y asincrónicas, evitando descargas innecesarias, escuchar música, reproducir videos o tener juegos online. Si se conecta desde el wifi del hogar, comunicar a los integrantes de la burbuja familiar, que durante ese tiempo se necesita todo el ancho de banda para el estudio.

Esto debe de ejecutarse, no solo con base en las escaseces pedagógicas que se logren presentar, en donde la mediación seguirá siendo indispensable; asimismo 
observando todos aquellos elementos que logran conjeturar una situación de desventaja; y es aquí donde corresponderá proporcionar un cuidado especial a las medidas forzosas para el reequilibrio geográfico, atando estas no solo a la metamorfosis y el desarrollo de las localidades, sino también, a la calidad de vida independientemente de la procedencia y al equilibrio social.

\section{Referencias}

Álvarez, D. (2020a, 23 de marzo). COVID-19, Educación digital y el futuro que se anticipa. E-aprendizaje. https://e-aprendizaje.es/2020/03/23/ covid-19-educacion-digital-y-el-futuro-que-se-anticipa/

Anaya, T., Montalvo, J., Calderón, A. I., y Arispe, C. (2021). Escuelas rurales en el Perú: factores que acentúan las brechas digitales en tiempos de pandemia (COVID- 19) y recomendaciones para reducirlas. Educación, 30(58), 11-33. https://doi.org/10.18800/ educacion.202101.001

Brenner, M. A. (2020, 4 de junio). Google classroom, la cuarentena. El silencio como ausencia pedagógica. Alainet. https://www.alainet.org/es/articulo/205718

Boursicot, K., Kemp, S., Ong, T. H., Wijaya, L., Goh, S. H., Freeman, K., y Curran, I. (2020). Conducting a high-stakes OSCE in a COVID-19 environment. MedEdPublish, 1-9. https://doi.org/10.15694/mep.2020.000054.1

Chanto, C., y Loáiciga, J. (2020). Educandos universitarios: entre la brecha digital y el aprendizaje en tiempos de COVID-19. El caso de Universidad Nacional de Costa Rica (UNA), Sede Región Chorotega, Campus Liberia. Revista Nuevo Humanismo, 8(2), 95-122. http://dx.doi.org/10.15359/rnh.8-2.5

Fondo de Naciones Unidas para la Infancia (2021, 22 de abril). Alfabetización digital para garantizar el presente y el futuro de la Generación del Bicentenario. https://www.unicef.org/costarica/comunicados-prensa/ alfabetizacion-digital-para-garantizar-el-presente-y-el-futuro-de-la-generacion

Gewin, V. (2020). Five tips for moving teaching online as COVID-19 takes hold. Nature, 580(7802), 295-296. https://doi.org/10.1038/d41586-020-00896-7

Gonzales-Zamora, J. A., Alave, J., De Lima-Corvino, D. F., y Fernández, A. (2020). Videoconferences of Infectious Diseases: An educational tool that transcends borders. A useful tool also for the current COVID-19 pandemic. Le infezioni in medicina, 28(2), 135. https://pubmed.ncbi.nlm.nih.gov/32275254/

Hodges, Ch., Moore, S., Lockee, B., Trust, T. y Bond, A. (2020, 27 de marzo). The Difference Between Emergency Remote Teaching and 
Online Learning. Educause Review. https://er.educause.edu/articles/2020/3/ the-difference-between-emergency-remote-teaching-and-online-learning

La Confederación Española de Asociaciones de Madres y Padres del Alumnado (2020, 30 de marzo). Las familias piden a los profesores "dosificar las excesivas tareas a distancia”. Público. https://www.publico.es/sociedad/familias-piden-profesores-dosificar-excesivas-tareas-distancia.html

Martínez, O. (2020). Brecha digital educativa. Cuando el territorio es importante. Sociedad e Infancias, 4, 267-270. https://doi.org/10.5209/soci.69629

Mian, A., y Khan, S. (2020). Medical education during pandemics: a UK perspective. BMC medicine, 18(1), 1-2. https://doi.org/10.1186/s12916-020-01577-y

Organización de las Naciones Unidas para la Educación, la Ciencia y la Cultura (2020a, 16 de marzo). Interrupción y respuesta educativa. https:/es.unesco.org/covid19/ educationresponse

Organización de las Naciones Unidas para la Educación, la Ciencia y la Cultura (2020b) COVID-19 y educación superior. De los efectos inmediatos al día después. Análisis de impactos, respuestas políticas y recomendaciones. [Archivo PDF]. http://www.iesalc. unesco.org/wp-content/uploads/2020/05/COVID-19-ES-130520.pdf

Plan Nacional de Desarrollo de las Telecomunicaciones (2015). Costa Rica: una sociedad conectada. [Archivo PDF]. https://www.micit.go.cr/sites/default/files/pndt-20152021_2.pdf

Programa Estado de la Nación (2020). Informe Estado de la Nación. [Archivo PDF]. http://www.conicit.go.cr/sic/biblioteca_virtual/publicaciones/publica_cyt/informes/ Informe_Estado_Nacion_2020.pdf

Torices, A. (2020, 31 de marzo). Educación distribuirá 20000 líneas móviles entre estudiantes sin medios telemáticos. Diario La Rioja. https://www.larioja.com/sociedad/ educacion-distribuira-20000-20200330175127-ntrc.html

Trujillo, F. (2020a 18 de marzo). Sentido común pedagógico frente a la crisis del coronavirus. Educación 3.0. https://www.educaciontrespuntocero.com/opinion/ exceso-de-deberes-para-los-estudiantes-coronavirus/

Trujillo, F. (2020b, 26 de marzo). Educación frente al coronavirus. Fernando Trujillo. https:/fernandotrujillo.es/ano-2020-educacion-frente-al-coronavirus/

Vallespín, I. (2020, 7 de abril). CC.OO. alerta de la brecha digital entre los profesores para impartir clases en línea. El País. https:/elpais.com/espana/catalunya/2020-04-07/ccoo-alerta-de-la-brecha-digital-entre-los-profesores-para-impartir-clases-en-linea.html 

Universities (UNA - UCR - UNED - UTN)

Vivanco-Saraguro, A. (2020). Teleducación en tiempos de COVID-19: brechas de desigualdad. CienciAmérica, 9(2), 166-175. http://dx.doi.org/10.33210/ca.v9i2.307

Wanderley, F., y Calvo, C. (2020). Educación y brecha digital. Trabajo de calidad. [Archivo PDF] https://boliviadebate.org/wp-content/uploads/2021/01/Cartilla-5.pdf

Wang, C., Cheng, Z., Yue, X.-G., y McAleer, M. (2020). Risk Management of COVID-19 by Universities in China. Journal of Risk and Financial Management, 13(2), 36. https:// doi.org/10.3390/jrfm13020036

Wendelboe, A. M., Miller, J. D., Drevets, D., Salinas, L., Miller, E. J., y Jackson, D. (2020). Tabletop exercise to prepare institutions of higher education for an outbreak of COVID-19. Journal of Emergency Management, 18(2), 183. https://doi.org/10.5055/ jem.2020.0463

Zhang, W., Wang, Y., Yang, L., y Wang, C. (2020). Suspending Classes Without Stopping Learning: China's Education Emergency Management Policy in the COVID-19 Outbreak. Journal of Risk and Financial Management, 13(3), 1-6. https://doi.org/10.3390/ jrfm13030055 